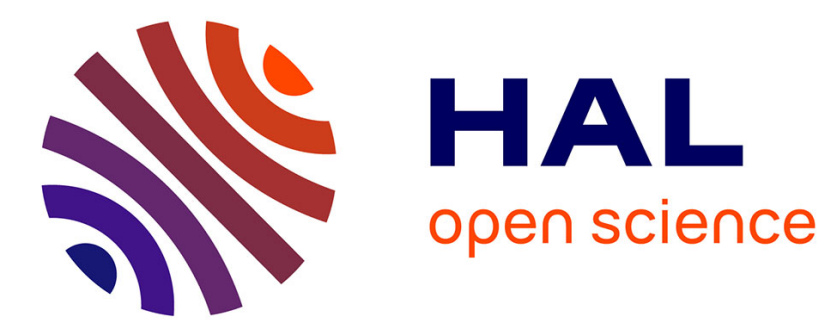

\title{
Resolving the activation mechanism of the D99N antiterminator LicT protein
}

Yinshan Yang, Jérôme Gracy, Nathalie Declerck, Héléne Démèné

\section{To cite this version:}

Yinshan Yang, Jérôme Gracy, Nathalie Declerck, Héléne Démèné. Resolving the activation mechanism of the D99N antiterminator LicT protein. Journal of Structural Biology, 2021, 213 (2), pp.107730. 10.1016/j.jsb.2021.107730 . hal-03088255

\section{HAL Id: hal-03088255 https://hal.science/hal-03088255}

Submitted on 25 Dec 2020

HAL is a multi-disciplinary open access archive for the deposit and dissemination of scientific research documents, whether they are published or not. The documents may come from teaching and research institutions in France or abroad, or from public or private research centers.
L'archive ouverte pluridisciplinaire HAL, est destinée au dépôt et à la diffusion de documents scientifiques de niveau recherche, publiés ou non, émanant des établissements d'enseignement et de recherche français ou étrangers, des laboratoires publics ou privés. 
Manuscript Number:

Title: Resolving the activation mechanism of the D99N antiterminator LicT protein.

Article Type: Structure Report

Keywords: NMR; BglG; antitermination protein; activation mechanism; signal transduction

Corresponding Author: Dr. Helene Demene, Ph. D.

Corresponding Author's Institution: CNRS 5048 INSERM U554, F34090 Montpellier, France; CNRS UMR5048, Université Montpellier 1 et 2, Centre de Biochimie Structurale, F34090 Montpellier, France;

First Author: Yinshan Yang, PhD

Order of Authors: Yinshan Yang, PhD; Jérome Gracy, PhD; Nathalie Declerck, PhD; Helene Demene, Ph. D.

Abstract: LicT is an antiterminator protein of the BglG family whose members are key players in the control of carbohydrate catabolism in bacteria. These antiterminators are generally composed of three modules, an N-terminal RNA-binding domain (CAT) followed by two homologous regulation modules ( $\mathrm{PRD} 1$ and $\mathrm{PRD} 2$ ) that control the RNA binding activity of the effector domain via phosphorylation on conserved histidines. Although several structures of isolated domains of BglG-like proteins have been described, no structure containing CAT and at least one PRD simultaneously has yet been reported in an active state, precluding detailed understanding of signal transduction between modules. To fulfill this gap, we recently reported the complete NMR sequence assignment of a constitutively active mutant (D99N) CAT-PRD1*, which contains the effector domain and the first regulation domain of LicT. As a follow-up, we have determined and report here the 3D solution structure of this active, dimeric LicT construct $(40 \mathrm{kDa})$. The structure reveals how the mutation constrains the PRD1 regulation domain into an active conformation which is transduced to CAT via a network of negatively charged residues belonging to PRD1 dimeric interface and to the linker region. In addition, our data support a model where BglG-type antitermination regulatory modules can only adopt a single conformation compatible with the active structure of the effector domain, regardless of whether activation is mediated by mutation on the first or second PRD. The linker between the effector and regulation modules appears to function as an adaptable hinge tuning the position of the functional modules.

Suggested Reviewers: Stefan Becker PhD

Group leader, Department of NMR-based Structural Biology, Max Planck Institute for Biophysical Chemistry

sabeenmr.mpibcp.mpg.de

Stefan Becker published a structure of an inactive fragment of a protein homolog. 
Sophie Zinn-Justin PhD

Group Leader, Institute for Integrative Biology of the Cell sophie.zinnecea.fr

Dr Sophie Zinn-Justin is an expert in NMR and X-ray structure of DNA binding proteins.

Walter J Chazin PhD

Group leader, Department of Biochemistry, Vanderbilt University

walter.chazin@vanderbilt.edu

Dr Walter Chazin is specialized in NMR studies of modular proteins. 


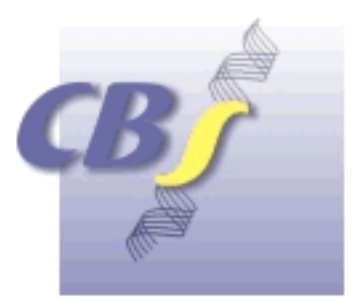

Centre

de

Biochimie

Structurale

CNRS-INSERM-UM1
Dr. Hélène Déméné

Tel : + 33 (0) 467417701

Fax : +33 (0) 4677913

e-mail : Helene.Demene@cbs.cnrs.fr

To Associate Editor,

Montpellier, December the 8 th

\section{Dear Editor,}

Please find enclosed a version of our manuscript, entitled "Resolving the activation mechanism of the D99N antiterminator LicT protein." LicT belongs to a family of bacterial transcriptional antiterminators, which control the expression of sugar-metabolizing operons in response to phosphorylations by the phosphoenolpyruvate:sugar phosphotransferase system (PTS). Like most proteins of this family, LicT is a dimeric protein composed of a RNA-binding module, whose antitermination activity is controlled by antagonist phosphorylation on the two regulation modules which module the dimeric interfaces. Several structures of the isolated domains in their active conformation have been solved so far (Yang et al. Embo J 2002; van Tillbeurgh Embo J 2001; Graille et al. J Biol Chem 2005), but none that contains an effector and a regulation domain simultaneously. We report here the full NMR characterization of D99N CAT-PRD1* LicT. This mutant fragment is active (Lindner et al. J Bact 2002) and contains the RNA-binding domain and the first regulation module of LicT. We identify a network of negatively charged residues which mechanically transduce the activation signal from the regulatory module to the effector module via the linker region in between. In addition, we demonstrate that the effector and regulatory modules adopt an active state independent of the activation mode (mutation in the first or second module) by contrast to the linker region, which tunes its conformation toward the functional output. Hence, we think our work is of broad interest for the scientific community interested in bacterial metabolism, but also more generally in the structure of modular proteins ensuring signal 
transduction. We therefore hope that our manuscript meets the standards of publication in Journal of

Structural Biology.

Sincerely yours,

Dr. Hélène Déméné.

Potential referees:

Stefan Becker sabe@nmr.mpibpc.mpg.de

Planck Institute for Biophysical Chemistry

Gottingen 37077, Germany

Sophie Zinn-Justin, sophie.zinn@cea.fr, Institute for Integrative Biology of the Cell (I2BC), CEA, CNRS, Université Paris-Sud, Université Paris-Saclay, Gif-sur-Yvette Cedex, France;

Walter J Chazin, walter.chazin@vanderbilt.edu

Department of Biochemistry, Vanderbilt University, Nashville, TN 37240-7917, USA 
Highlights:

- First NMR structure of an active LicT fragment containing effector and regulation modules.

- The structure of modules is independent of the activation mode.

- Identification of a network of negatively charged residues which relay signal between modules.

- The linker region adapts its structure to produce the functional output. 


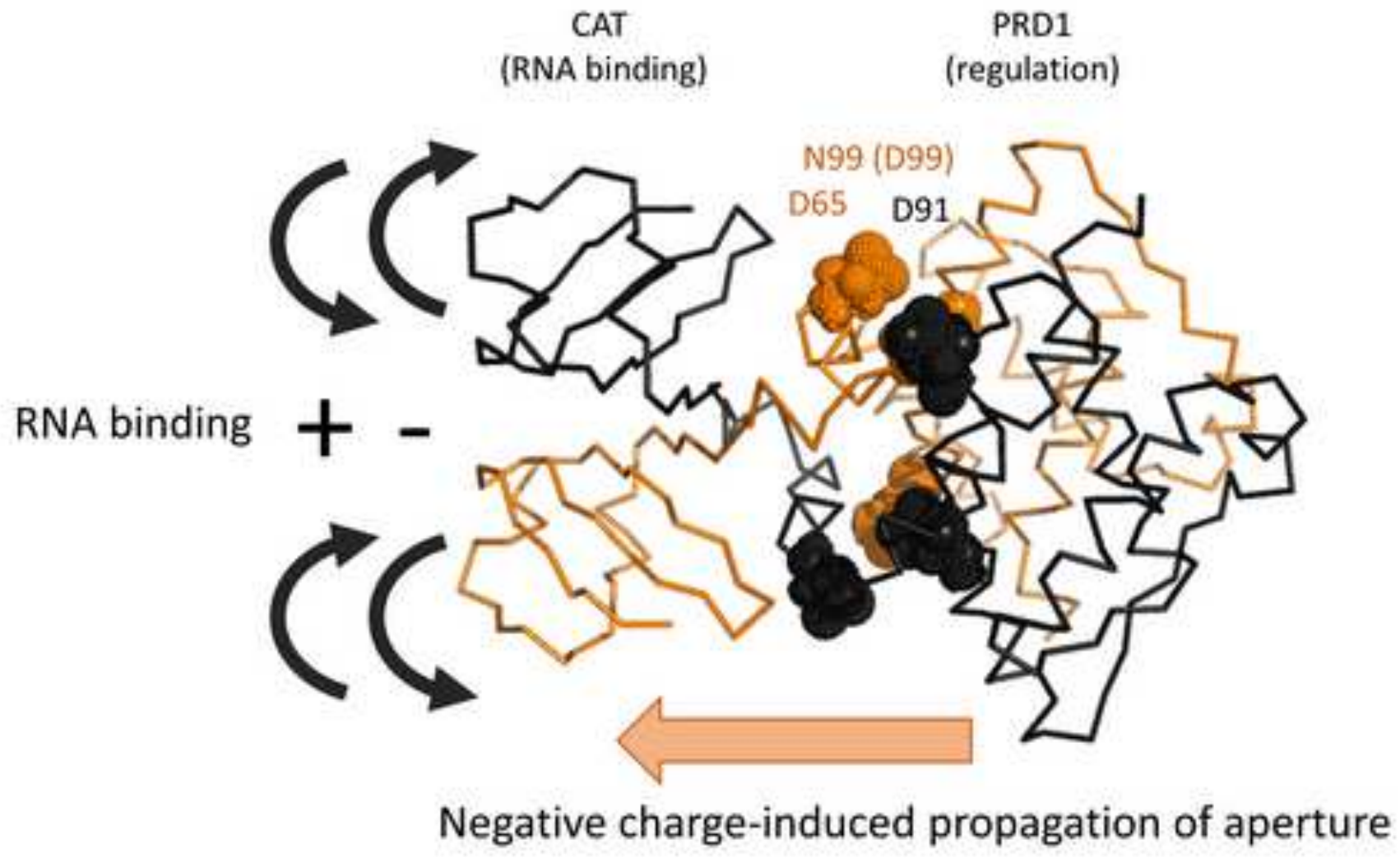


Resolving the activation mechanism of the D99N antiterminator LicT protein.

Corresponding author: Hélène Déméné: helene.demene@cbs.cnrs.fr

Declarations of interest: none

\begin{abstract}
LicT is an antiterminator protein of the BglG family whose members are key players in the control of carbohydrate catabolism in bacteria. These antiterminators are generally composed of three modules, an N-terminal RNA-binding domain (CAT) followed by two homologous regulation modules (PRD1 and PRD2) that control the RNA binding activity of the effector domain via phosphorylation on conserved histidines. Although several structures of isolated domains of BglG-like proteins have been described, no structure containing CAT and at least one PRD simultaneously has yet been reported in an active state, precluding detailed understanding of signal transduction between modules. To fulfill this gap, we recently reported the complete NMR sequence assignment of a constitutively active mutant (D99N) CAT-PRD1*, which contains the effector domain and the first regulation domain of LicT. As a follow-up, we have determined and report here the 3D solution structure of this active, dimeric LicT construct $(40 \mathrm{kDa})$. The structure reveals how the mutation constrains the PRD1 regulation domain into an active conformation which is transduced to CAT via a network of negatively charged residues belonging to PRD1 dimeric interface and to the linker region. In addition, our data support a model where BglG-type antitermination regulatory modules can only adopt a single conformation compatible with the active structure of the effector domain, regardless of whether activation is mediated by mutation on the first or second PRD. The linker between the effector and
\end{abstract}


regulation modules appears to function as an adaptable hinge tuning the position of the functional modules.

Keywords: NMR ; BglG; antitermination protein; activating mutation; signal transduction.

Authors contribution

Yinshan Yang: Investigation and Visualization. Jérome Gracy: Visualization. Nathalie Declerck: Investigation, Supervision and Writing- review and editing; Hélène Déméné: Investigation, Supervision, Writing- Original Draft writing, review and editing. 
The ability of signaling proteins to switch between active and inactive conformations constitutes the molecular basis of signal transduction. Phosphorylation plays a prominent role in toggle events for modular proteins. This is the case for antiterminator proteins of the BglG family, whose RNA binding capacity is governed by phosphorylation-based signals on regulation modules propagated to a distant effector module (Mahadevan and Wright, 1987; Van Tilbeurgh and Declerck, 2001). BglG-like antiterminators control the catabolism of carbohydrate substrates by binding to a stpecific sequence of RNA messenger of sugar operons. Antiterminators of this protein family are typically composed of three domains: an RNA-binding domain named CAT (for co anti-terminator), and two homologous regulation domains. The isolated CAT domain is constitutively active, but in the whole protein, its RNA binding affinity is controlled by antagonist phosphorylation by phosphotransferase (PTS) enzymes on conserved histidines in the regulation modules, named PRD1 and PRD2 (for PTS regulation domain) (Stülke et al., 1998). Bacillus subtilis LicT represents a prototypical BglG-type antiterminator in Gram positive bacteria, which governs assimilation of complex sugar such as aryl$\beta$-glucosides (Schnetz et al., 1996). The structure of several LicT fragments have been solved, either by NMR or X-ray crystallography (Fig. 1A). The structure of the isolated CAT domain solved by NMR revealed a folding in a symmetrical $\beta$-sheet sandwich that remains unchanged upon binding to RNA with a 2:1 stoichiometry (Yang et al., 2002). It was shown that LicT can reach a constitutive active state (LicT*) through phosphomimetic mutations changing the phosphorylatable histidine residues of PRD2 (H207 and H269) into aspartic acids (Declerck et al., 2001; Tortosa et al., 2001). Comparison of crystallographic structures of the LicT regulatory domains in the native (PRD1-PRD2) and active (PRD1-PRD2*) states revealed that activating mutations promoted the closing of a loose dimer into a compact topology, keeping individual protomer structures intact (Marc Graille et al., 2005; van Tilbeurgh et al., 2001).

For a long time, structural information has been lacking on the mechanism of signal transduction between effector and regulation modules of these antitermination proteins. A model of CAT-PRD1 containing the RNA binding domain and the first regulation domain from native LicT was proposed on the basis of NMR residual dipolar couplings and ${ }^{15} \mathrm{~N}$ relaxation measurements, suggesting an opening of the CAT dimer in the inactive state (Déméné et al., 2008). We recently confirmed this hypothesis by a NMR systematic study on truncated and full-length proteins, either in their native or activated states, using ${ }^{13} \mathrm{C}$-labeled methionines as markers of topology of the different dimers (Yang et al., 2020b). Although several 3D structures of LicT fragments have been reported (Fig. 1A) (M Graille et al., 2005; van Tilbeurgh et al., 2001; Yang et al., 2002), no structure at atomic resolution has ever been solved that shows the RNA-binding domain linked to a regulation module of this protein in a 
RNA-binding competent conformation. Here we succeeded at determining the NMR solution structure of an activated form of the CAT-PRD1 bi-module, providing a comprehensive view of LicT activation mechanism. We took advantage of the existence of a point mutation in PRD1 (D99N) that renders LicT constitutively active in B. subtilis (Lindner et al., 2002) and that enhances in vitro stability and RNA-binding when introduced into the CAT-PRD1 bi-module (Demene 2008). The NMR structure of this CAT-PRD1* mutant illuminates a critical network of amino acids involved in the signal transduction between the PRD1 and CAT modules and fulfills a gap of knowledge in BglG-like antiterminators.

\section{Material and methods}

\subsection{Protein expression and purification.}

The CAT-PRD1* bi-module from LicT* (residues 1-167) carrying the D99N activating mutation (Lindner et al., 2002) was expressed as histidine-tagged proteins in E. coli BL21(DE3), either in rich LB medium, or in M9 minimal medium, supplemented with ${ }^{15} \mathrm{NH}_{4} \mathrm{Cl}$ and ${ }^{13} \mathrm{C}$-D-glucose as the sole nitrogen and carbon sources for ${ }^{15} \mathrm{~N}$ and ${ }^{13} \mathrm{C}$ uniform labelling. Methionine- $\varepsilon_{-}^{-13} \mathrm{CH}_{3}$ (Sigma) at a 250 mg. $\mathrm{L}^{-1}$ concentration was added 20 minutes before 2 hours induction at $37^{\circ} \mathrm{C}$ for specific methionine labelling. Otherwise, induction was provoked by addition of $0.5 \mathrm{mM}$ isopropyl- $\beta$-Dthiogalactopyranoside (IPTG) at $20^{\circ} \mathrm{C}$ for 20 hours. Purification was performed by a step of nickel chromatography followed by size exclusion chromatography in NMR buffer, as described elsewhere (Yang et al., 2020a).

\subsection{NMR assignment and structure calculations.}

CAT-PRD1* NMR assignment has been already described (Yang et al., 2020a). Briefly, it was obtained by double and triple 3D resonance experiments HNCO, HNCA, CBCA(CO)NH, HNCACB, HCCH-total correlated spectroscopy, ${ }^{15} \mathrm{~N}$ edited HSQC-NOESY, and ${ }^{13}$ Cedited HSQC-NOESY. Experiments were performed at $308 \mathrm{~K}$ to increase sensitivity. For detection of intermolecular NOEs, a differentially labelled sample was prepared by mixing equimolar amounts of ${ }^{15 \mathrm{~N}}$-labelled CAT-PRD1 and ${ }^{15} \mathrm{~N} /{ }^{13} \mathrm{C}$-labelled CAT-PRD1 at a concentration of $2 \mathrm{mg} / \mathrm{ml}$ in $6 \mathrm{M}$ urea. The sample was then dialyzed $2 \times 3$ hours against the NMR buffer (200 mM NaCl, pH 8, $10 \mathrm{mM}$ Tris, $0.5 \mathrm{mM}$ EDTA, 0.2 $\mathrm{mM}$ benzamidine, $1 \mathrm{mM}$ DTT) and concentrated to a final concentration of $600 \mu \mathrm{M}$. After lyophylization, the protein was resuspended in $\mathrm{D}_{2} \mathrm{O}$ and introduced into an oval-shaped NMR tube (Bruker Biospin) optimized for highly salted samples. This sample enabled the collection of additional $150(75 \times 2)$ intermolecular distance constraints from a $2 \mathrm{D}$ version of a 3D F1-13C-filtered/F3- ${ }^{13} \mathrm{C}$ edited NOESYHSQC spectrum $(\tau \mathrm{mix}=100 \mathrm{~ms}) .145$ backbone $\phi$ and $\psi$ and $83 \chi^{2} / \chi^{3}$ dihedral angle constraints were generated per monomer from secondary shifts of the ${ }^{1} \mathrm{H} \alpha,{ }^{13} \mathrm{C} \alpha,{ }^{13} \mathrm{C} \beta,{ }^{13} \mathrm{C}^{\prime}$, and ${ }^{15} \mathrm{~N}$ nuclei shifts using the TALOS software (Shen and Bax, 2015). Initial structures were generated with 
the CYANA software (Güntert and Buchner, 2015) using a set of NOE unambiguously assigned. The characterized in the preliminary structures and supplementary disambiguated NOEs, in subsequent rounds of CYANA. At the final stage, a total of 1850 distance constraints for each monomer was used (Table 1). Of the 100 CYANA structures calculated, the 20 conformers with the lowest target function were subjected to a minimization protocol using Xplor (Brüner et al., 1998). The coordinates of the 20 resulting structures and the NMR restraint set were deposited in the PDB under entry 6TWR.

\section{Results and discussion}

\subsection{Comparison of PRD1 configuration in active states by NMR}

In a previous study, we used NMR chemical shifts of the RNA binding domain CAT to establish its structure conservation across all active LicT fragments, including CAT-PRD1*, except for marginal differences at the dimer interface (Yang et al., 2020b). We have now performed the full assignment of CAT-PRD1* resonance peaks (Yang et al., 2020a), and we can now analyze the configuration of the PRD1 module in this active state as well. The NMR peaks of the PRD1 module are slightly different between active CAT-PRD1* and LicT*, suggesting a difference in PRD1 conformation between both proteins (Fig. 1B). This difference was unexpected, since by contrast and as already mentioned (Yang et al., 2020b), PRD1 resonances were remarkably well conserved between PRD1-PRD2* and LicT* (Fig. 1C). These differences could reach the value of $1 \mathrm{ppm}$, as for D90 which lies in front of the side chain of the mutated N99 of the other protomer (Fig. S1). Their mapping on the model structure of full length activated LicT* (Yang et al., 2020b) indicates a localization at the PRD1 dimeric interface (Fig. 1D).

\subsection{NMR structure of CAT-PRD1*}

Previous analysis by size exclusion chromatography and analytical ultracentrifugation revealed that CAT-PRD1* is dimeric in solution, at concentration as low as $50 \mu \mathrm{M}$ (Déméné et al., 2008). The observation of a single set of resonances for CAT-PRD1* (Fig.1B) indicates that the dimer adopts a symmetric fold. To decipher its architecture, we have recorded a $\mathrm{F} 1-{ }^{13} \mathrm{C}$-filtered, $\mathrm{F} 3-{ }^{13} \mathrm{C}$ edited HSQC NOESY experiment on a sample containing an equimolar amount of ${ }^{15} \mathrm{~N} /{ }^{13} \mathrm{C}$-labelled/unlabelled proteins. Fig. 2A depicts a portion of the doubly filtered NOESY spectrum showing inter-molecular correlations between aromatic/aliphatic protons. We could assign 75 (x2) intermolecular NOEs, of which 33 and 42 involved the CAT and PRD1 domains respectively. Added to intramolecular NOEs and hydrogen bonds, we obtained a final set of 1850 distance restraints, which we complemented by 228 dihedral restraints derived from chemical shifts. A final ensemble of 20 structures is presented in Fig. 2B, and structural statistics are presented in Table 1. 


\subsection{CAT and PRD1 conserve their individual monomeric structure in CAT-PRD1* but the dimer}

conformation is slightly changed.

Backbone RMSD values of $1.1 \pm 0.1 / \AA$ and $1.1 \pm 0,1 \AA$ were determined for the individual monomers and the dimers of CAT-PRD1* (Table 1). The perfect agreement of the backbone RMSD values between monomers and dimers indicates that the dimer interface is highly determined, allowing for the proper orientation of the two halves. Comparison of the RMSD values for the CAT and PRD1 domain alone reveals that the structure of the CAT dimeric domain is marginally better defined than the PRD1 dimeric domain (RMSD of 0.7 versus $1.0 \AA$ ).

In CAT-PRD1*, CAT monomer adopts the previously documented $\beta 1 \beta 2 \beta 3 \beta 4$ topology composed of four antiparallel $\beta$-strands and a long loop connecting strands 3 and 4 (Declerck et al., 1999; Yang et al., 2002). The PRD1 domain harbors the classical five-helix bundle conformation which was observed for native and activated PRD1-PRD2 bi-modules (Marc Graille et al., 2005; van Tilbeurgh et al., 2001). We proceeded to a thorough comparison of the structure of CAT-PRD1* modules with the different available structures of CAT and PRD1-PRD2*. The overall structure of the monomeric form of CAT and PRD1 domains was conserved, with an RMSD of $1.13 \pm 0,07 \AA$ for CAT (comparison structure 1L1C) and 1.35 $\pm 0,07 \AA$ for PRD1 (comparison structure 1H99) respectively.

We then checked the dimer topology for each module of CAT-PRD1* and found that they were also overall well conserved. However, we found slight but significant differences, highlighted by a higher RMSD against the reference structure than the RMSD within the NMR ensemble, namely $1.2 \pm 0.1 \AA$ versus $0.76 \pm 0.07 \AA$ and $1.7 \pm 0.1 \AA$ versus $1.1 \pm 0.10 \AA$ for CAT and PRD1 respectively. These differences correspond to a higher and smaller aperture of dimers in CAT and PRD1 respectively (Figs. 3A-B) and are confirmed by the differences in chemical shifts observed at the respective dimer interfaces (Yang et al., 2020b) and Fig. 1D).

\subsection{Active structure of CAT is mediated by a different architecture of the linker region in CAT- PRD1* and LicT*.}

It was previously postulated, on the basis of limited proteolysis and circular dichroism data, that the linker region joining CAT to PRD1 (residues 52-68) was disordered in active CAT-PRD1*, enabling CAT dimer closure, whereas its folding into a continuous helix leads to CAT dimer opening in inactive CAT-PRD1 (Déméné et al., 2008). This hypothesis was supported by the observation of a disordered structure at the N-terminal end of the crystallized PRD1-PRD2* bi-module (residues 57277) bearing phosphomimetic activating mutations in the PRD2 module (van Tilbeurgh et al., 2001) whereas a succession of turns was observed for the native PRD1-PRD2 fragment (Marc Graille et al., 2005). The linker folded also as an helix in the structure of the native (inactive) CAT-PRD1 domain of 
the GlcT homolog, which crystallized as a monomer (Himmel et al., 2012). Having solved the first aspect of signal transduction between effector and regulation modules. Unexpectedly, the linker in CAT-PRD1* is not disordered, but folds as a helix between residues F59 and I65, preceded by a turn at the level of D54 linking this helix to the $\beta 5$ sheet at the CAT dimeric interface (Fig. 3C). Despite the difference in linker structures between CAT-PRD1* and PRD1-PRD2*, their extremities are found to superpose surprisingly well; in particular, the first residue of the linker, N52 of CAT-PRD1*, is found at the same spatial location of G54 of PRD1-PRD2* (Fig. 3C). However, PRD1-PRD2* has a $\mathrm{N}$-terminal hexahistidine tag which alters the native sequence from $\mathrm{N}^{52} \mathrm{KDVSE}^{57}$ to $\mathrm{G}^{54} \mathrm{SME}^{57}$.

To check if these differences in linker conformation were related to crystal packing strains in the crystal structure of PRD1-PRD2* or difference in sequence due its $\mathrm{N}$-terminal tag, we turned to the comparison of NMR chemical shifts between CAT-PRD1*, PRD1-PRD2* and LicT* in solution. The perfect superimposition of PRD1-PRD2 ${ }^{1} \mathrm{H}-{ }^{15} \mathrm{~N}$ correlation peaks with those of LicT* in ${ }^{1} \mathrm{H}^{-15} \mathrm{~N}$ HSQC spectra (Fig. 1B) and the differences with those of CAT-PRD1* (Figs. 1 C-D and S1) suggests a conservation of linker structure in PRD1-PRD2* and in LicT*, in contrast to CAT-PRD1*. We previously showed that LicT methionine methyl groups are useful NMR markers of LicT dimer topology (Yang et al., 2020b). We thus checked NMR chemical shifts of M71, which is at located just at the beginning of PRD1 fragment, next to the linker. Comparison of NMR spectra of ${ }^{13} \mathrm{C}$ methyl labelled-methionine LicT fragments confirms that M71 methyl groups experience a very different chemical shift environment between CAT-PRD1* on one side and PRD1-PRD2* and LicT* on the other side (Fig. 3D), presumably due to a change in orientation of the nearby side chain of F59 belonging to the linker (Fig.S2).

Hence, PRD1 dimeric configuration is found remarkably similar in all active states, whereas the linker conformation can adopt multiple conformations in order to accommodate activating mutations (or phosphorylation) in PRD1 or PRD2

\subsection{Mechanism of signal transduction from PRD1 to CAT in mutant CAT-PRD1*.}

Having solved the structure of active CAT-PRD1* enables a close comparison of the dimeric topology between CAT-PRD1* and inactive PRD1-PRD2 to understand the mutational effect. Figure 3D shows that the D99N mutation abolishes the repulsion network between three different charged residues in the native state that maintains PRD1 and the linker in an inactive open conformation. This repulsive network involves the side chains of D91 (PRD1 of A protomer), D99 (PRD1 of B protomer) and D65 (linker of $\mathrm{B}$ protomer) in the native state. Its disruption leads to the compaction of the dimeric interface, which was loose in the inactive state and which is now stabilized by intermolecular hydrogen bonds. Among the twenty CAT-PRD1* NMR conformers, we observe indeed a hydrogen 
bond between N99 side chain amide nitrogen atoms of one protomer and either the oxygen atoms of protomer, the latter D99-N90 bond being also present in the crystal structure of PRD1-PRD2*.

\subsection{Discussion and conclusion}

Prior to this study, active states of BglG-like antiterminator proteins whose structure was solved at atomic resolution, were the isolated CAT domains (X Manival et al., 1997; Xavier Manival et al., 1997) and the bi-module PRD1-PRD2* (van Tilbeurgh et al., 2001). Information was thus missing on the linker structure in an active state. We report here the first characterization at atomic resolution of an active fragment containing simultaneously an effector and a regulation domain, namely the active CAT-PRD1*. Its activation is obtained by the D99N mutation in the PRD1 domain. This mechanism is different from classical phosphomimetic mutations occurring in the PRD2 domain previously found to be equivalent to true phosphorylation by PTS enzymes (Yang et al., 2020b). We however observe that the CAT and PRD1 dimeric topologies in CAT-PRD1* exhibit only little difference with the active structures solved so far. This unicity of active conformation at the level of the effector and regulation domain of LicT is remarkable. It stands in contradiction with for example the regulation model reported for the regulator RegA. In the latter case, the conformations of the structure and dynamics of receiver domains were found to be distinct between activated states induced by mutation or by phosphorylation (Moorthy and Anand, 2012). More precisely, the structural data indicated that the activating F262W mutation induced the existence of an ensemble of multiple conformations, encompassing active and inactive states. By contrast, LicT activation seems to obey a scheme whose rigidity meets the requirements of transcription regulation dictated by bacterial survival. In addition, we establish that signal transduction involves a network of negatively charged residues connecting the PRD1 interface to the linker dimeric structure, which in turns governs CAT opening and RNA binding capacity. Contrary to CAT and PRD1 structures, the structure of the linker was different between all the active states, pointing out to this region as a major adaptive player in signal transduction.

\section{Accession numbers}

The coordinates and NMR restraints for active D99N CAT-PRD1* have been deposited in the Protein Data Bank (PDB) (https://www.rcsb.org/) with accession number 6TWR.

\section{Declaration of interest}

None. 


\section{Figure legends}

Fig. 1. NMR parameters of LicT fragments. (A) Schematic organization of active and inactive LicT constructs studied so far in the literature. NMR studies led to high resolution structures only for CAT (Yang et al., 2002) and CAT-PRD1* (this study). (B-C) Superposition of ${ }^{1} \mathrm{H}^{15}{ }^{15} \mathrm{~N}$ HSQC spectra of activated full-length H207D/H269D LicT* (red) and (B) H207D/H269D PRD1-PRD2* (black) or (C) D99N CAT-PRD1* (black). Correlation peaks (labelled with one letter code and residue number) which differ between CAT-PRD1* and LicT* is indicated by lines pointing to their position in CATPRD1*. (D) Structural model of activated full-length LicT* (Yang et al., 2020b), showing the position of $(\mathrm{H}, \mathrm{N})$ amide couples (red spheres) belonging to the dimer interface (left) or experiencing a shift exceeding $0.1 \mathrm{ppm}$ between D99N CAT-PRD1* and LicT* (right). An $(\mathrm{H}, \mathrm{N})$ amide couple is identified as part of the dimer interface when the $\mathrm{H}$ or $\mathrm{N}$ atom is within $5 \AA$ of any atoms of the other protomer. For clarity, data are represented only for one protomer.

Fig. 2. NMR derived structure of CAT-PRD1*

(A) Superposition of portions from the double half-filtered NOESY spectrum recorded on a ${ }^{13} \mathrm{C}$ ${ }^{15} \mathrm{~N}$ CAT-PRD1*/CAT-PRD1* sample (red) and from a regular NOESY recorded on non-labelled CAT-PRD1* (black). The superposition highlights intermolecular NOEs (red) between aromatic protons and aliphatic protons. Mixing time was 100 ms. (B) Ensemble of 20 final NMR conformers shown in ribbon representation. Superposition was performed taking backbone atoms of residues 3164.

Fig. 3. Close-up of CAT-PRD1* structural features. (A) CAT module (B) PRD1 module (C) linker region. For panels A-C, previously determined 3D structures (1L1C for CAT, 1H99 for PRD1PRD2*) are shown in magenta. The CAT and PRD1 modules conserve their monomeric conformations, although their dimeric structures exhibit slightly different degrees of aperture in solution compared to those observed in the crystals or in complex with RNA. The conformation of the linker region is by contrast different between CAT-PRD1* and PRD1-PRD2*, but seems to bring its extremities in similar positions compatible with CAT closure. (D) Comparison of M71 methyl chemical shifts between CAT-PRD1*, PRD1-PRD2* and LicT* suggest that the linker region in CATPRD1* has a different structure from that of PRD1-PRD2* and LicT*. (E) Highlight of the network of negatively charged residues (D65/D91/D99 shown as spheres) impacted by the D99N mutation at the inactive PRD1 interface (left), leading to closure of PRD1 domain and reorientation of the linker in CAT-PRD1* (right). 


\section{Acknowledgments}

The CBS is a member of the French Infrastructure for Integrated Structural Biology (FRISBI), supported by the National Research Agency (ANR-10- INBS-05) and is a GIS-IBIsA platform.

\section{References}

Brünger, A.T., Adams, P.D., Clore, G.M., Delano, W.L., Gros, P., Grossekunstleve, R.W., Jiang, J.S., Kuszewski, J., Nilges, M., Pannu, N.S., Read, R.J., Rice, L.M., Simonson, T., Warren, G.L., 1998. Crystallography \& NMR system: A new software suite for macromolecular structure determination. Acta Crystallogr. Sect. D Biol. Crystallogr. 54, 905-921. https://doi.org/10.1107/S0907444998003254

Declerck, N., Dutartre, H., Receveur, V., Dubois, V., Royer, C., Aymerich, S., Van Tilbeurgh, H., 2001. Dimer stabilization upon activation of the transcriptional antiterminator LicT. J. Mol. Biol. 314, 671-681. https://doi.org/10.1006/jmbi.2001.5185

Declerck, N., Vincent, F., Hoh, F., Aymerich, S., Van Tilbeurgh, H., 1999. RNA recognition by transcriptional antiterminators of the BgIG/SacY family: Functional and structural comparison of the CAT domain from SacY and LicT. J. Mol. Biol. 294, 389-402. https://doi.org/10.1006/jmbi.1999.3256

Déméné, H., Ducat, T., De Guillen, K., Birck, C., Aymerich, S., Kochoyan, M., Declerck, N., 2008. Structural Mechanism of Signal Transduction between the RNA-binding Domain and the Phosphotransferase System Regulation Domain of the LicT Antiterminator. J. Biol. Chem. 283, 30838-30849. https://doi.org/10.1074/jbc.M805955200

Graille, Marc, Zhou, C.-Z., Receveur-Bréchot, V., Collinet, B., Declerck, N., van Tilbeurgh, H., 2005. Activation of the LicT Transcriptional Antiterminator Involves a Domain Swing/Lock Mechanism Provoking Massive Structural Changes. J. Biol. Chem. 280, 14780-14789. https://doi.org/10.1074/jbc.m414642200

Graille, M, Zhou, C.Z., Receveur-Brechot, V., Collinet, B., Declerck, N., van Tilbeurgh, H., 2005. Activation of the LicT transcriptional antiterminator involves a domain swing/lock mechanism 
provoking massive structural changes. J Biol Chem 280, 14780-14789.

Güntert, P., Buchner, L., 2015. Combined automated NOE assignment and structure calculation with CYANA. J. Biomol. NMR 62, 453-471. https://doi.org/10.1007/s10858-015-9924-9

Himmel, S., Grosse, C., Wolff, S., Schwiegk, C., Becker, S., 2012. Structure of the RBD-PRDI fragment of the antiterminator protein GlcT. Acta Crystallogr. Sect. F Struct. Biol. Cryst. Commun. 68, 751-756. https://doi.org/10.1107/S1744309112020635

Lindner, C., Hecker, M., Le Coq, D., Deutscher, J., 2002. Bacillus subtilis mutant LicT antiterminators exhibiting enzyme I- and HPr-independent antitermination affect catabolite repression of the bglPH operon. J. Bacteriol. 184, 4819-4828. https://doi.org/10.1128/JB.184.17.4819-4828.2002

Mahadevan, S., Wright, A., 1987. A bacterial gene involved in transcription antitermination: Regulation at a rho-independent terminator in the bgl operon of E. coli. Cell 50, 485-494. https://doi.org/10.1016/0092-8674(87)90502-2

Manival, X, Aymerich, S., Strub, M.P., Dumas, C., Kochoyan, M., van Tilbeurgh, H., 1997. Crystallization of the RNA-binding domain of the transcriptional antiterminator protein SacY from Bacillus subtilis. Proteins 28, 590-4.

Manival, Xavier, Yang, Y., Strub, M.P., Kochoyan, M., Steinmetz, M., Aymerich, S., 1997. From genetic to structural characterization of a new class of RNA-binding domain within the SacY/BgIG family of antiterminator proteins. EMBO J. 16, 5019-5029. https://doi.org/10.1093/emboj/16.16.5019

Moorthy, B.S., Anand, G.S., 2012. Multistate allostery in response regulators: Phosphorylation and mutagenesis activate RegA via alternate modes. J. Mol. Biol. 417, 468-487. https://doi.org/10.1016/j.jmb.2012.01.052

Schnetz, K., Stülke, J., Gertz, S., Krüger, S., Krieg, M., Hecker, M., Rak, B., 1996. LicT, a Bacillus subtilis transcriptional antiterminator protein of the BglG family. J. Bacteriol. 178, 1971-9.

Shen, Y., Bax, A., 2015. Protein Structural Information Derived from NMR Chemical Shift with the Neural Network Program TALOS-N. pp. 17-32. https://doi.org/10.1007/978-1-4939-2239-0_2

Stülke, J., Arnaud, M., Rapoport, G., Martin-Verstraete, I., 1998. PRD--a protein domain involved in PTS-dependent induction and carbon catabolite repression of catabolic operons in bacteria. Mol. Microbiol. 28, 865-74.

Tortosa, P., Declerck, N., Dutartre, H., Lindner, C., Deutscher, J., Coq, D. Le, 2001. Sites of positive 
and negative regulation in the Bacillus subtilis antiterminators LicT and SacY. Mol. Microbiol. 41, 1381-1393. https://doi.org/10.1046/j.1365-2958.2001.02608.x

Van Tilbeurgh, H., Declerck, N., 2001. Structural insights into the regulation of bacterial signalling proteins containing PRDs. Curr. Opin. Struct. Biol. 11, 685-693. https://doi.org/10.1016/S0959440X(01)00267-6

van Tilbeurgh, H., Le Coq, D., Declerck, N., 2001. Crystal structure of an activated form of the PTS regulation domain from the LicT transcriptional antiterminator. EMBO J. 20, 3789-3799. https://doi.org/10.1093/emboj/20.14.3789

Yang, Y., Declerck, N., Déméné, H., 2020a. NMR chemical shift assignment of a constitutively active fragment of the antitermination protein LicT. Biomol. NMR Assign. 14, 19-23. https://doi.org/10.1007/s12104-019-09914-w

Yang, Y., Declerck, N., Manival, X., Aymerich, S., Kochoyan, M., 2002. Solution structure of the LicT-RNA antitermination complex: CAT clamping RAT. EMBO J. 21, 1987-1997. https://doi.org/10.1093/emboj/21.8.1987

Yang, Y., Guillen, K. De, Guillen, K. De, 2020b. Short Article Structural Insights into of the Allosteric Activation of the LicT Antiterminator by PTS-Mediated Short Article Structural Insights into of the Allosteric Activation of the LicT Antiterminator by PTS-Mediated Phosphorylation 1-8. https://doi.org/10.1016/j.str.2019.10.017 


\section{Figure}

Click here to download high resolution image

A

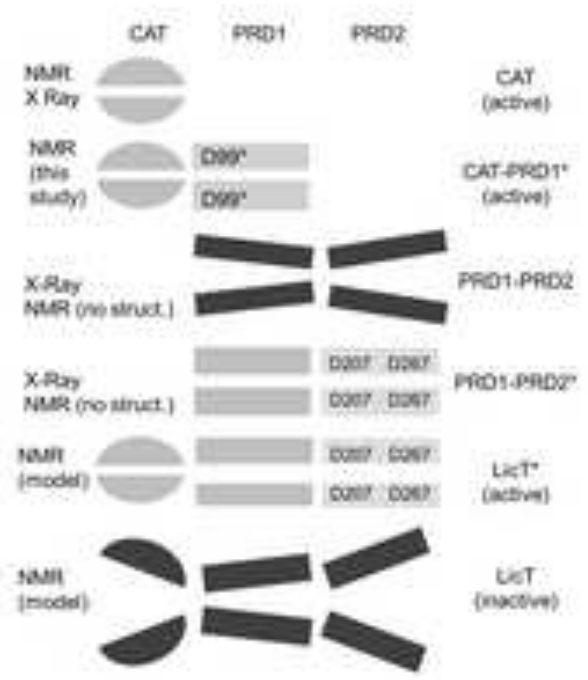

C

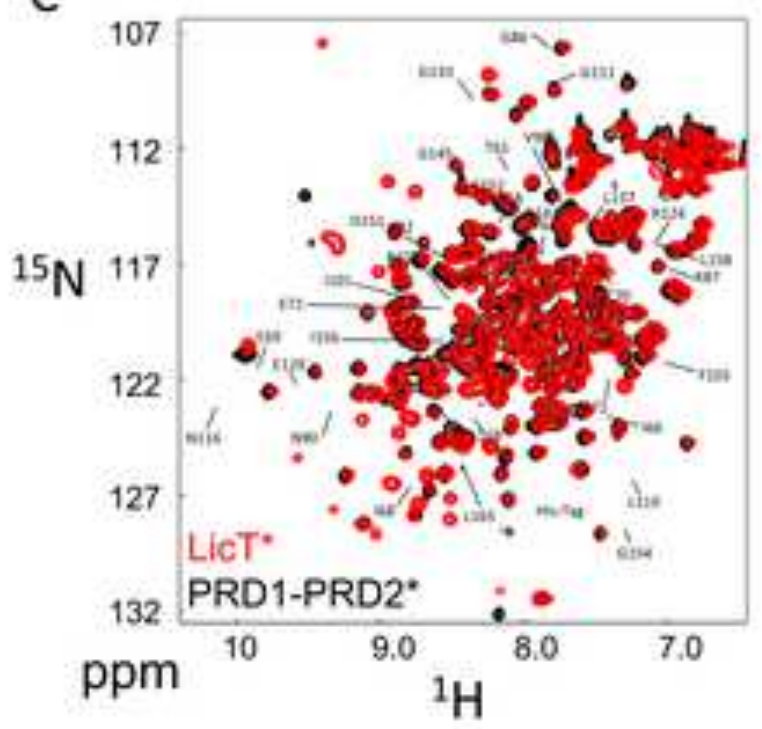

B

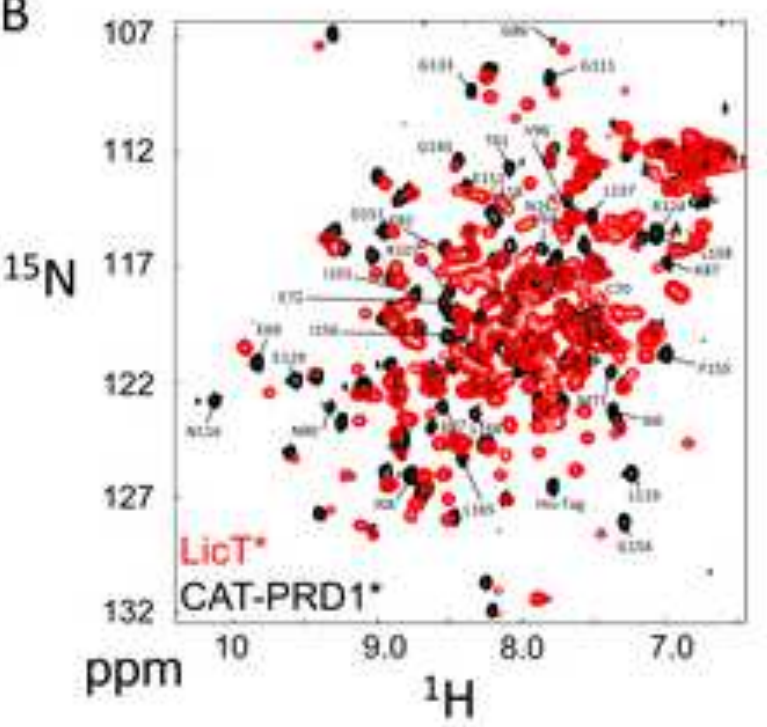

D

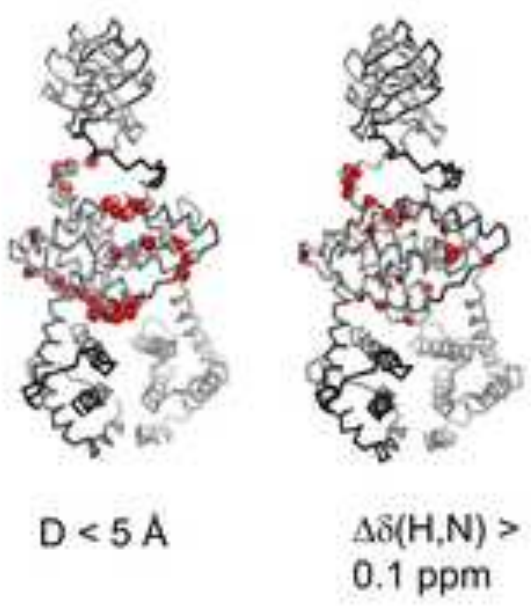

Fig.1

Yang et al. 


\section{Figure}

Click here to download high resolution image

A

\section{B}
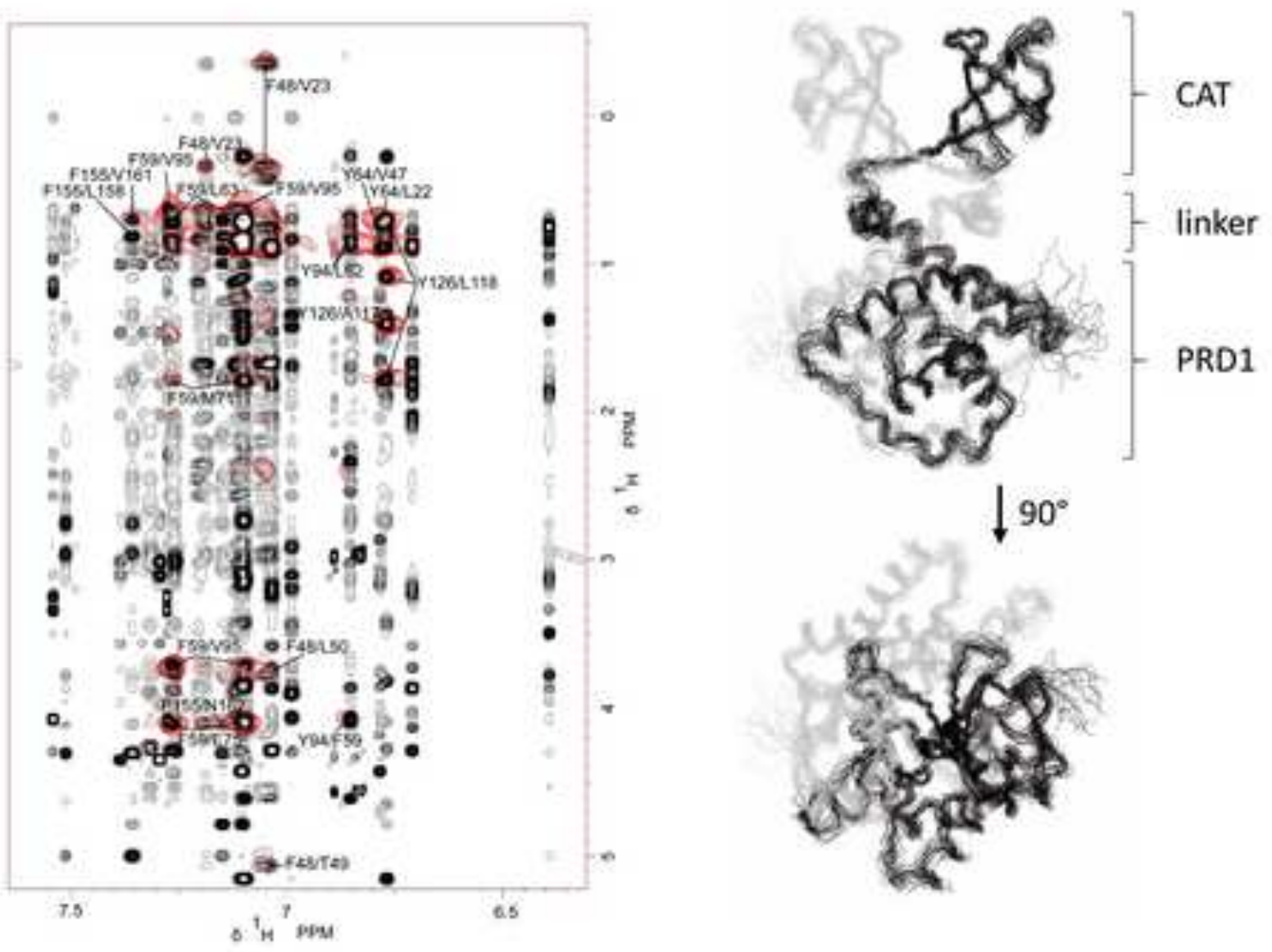

Figure 2

Yang et al. 
A

\section{CAT}
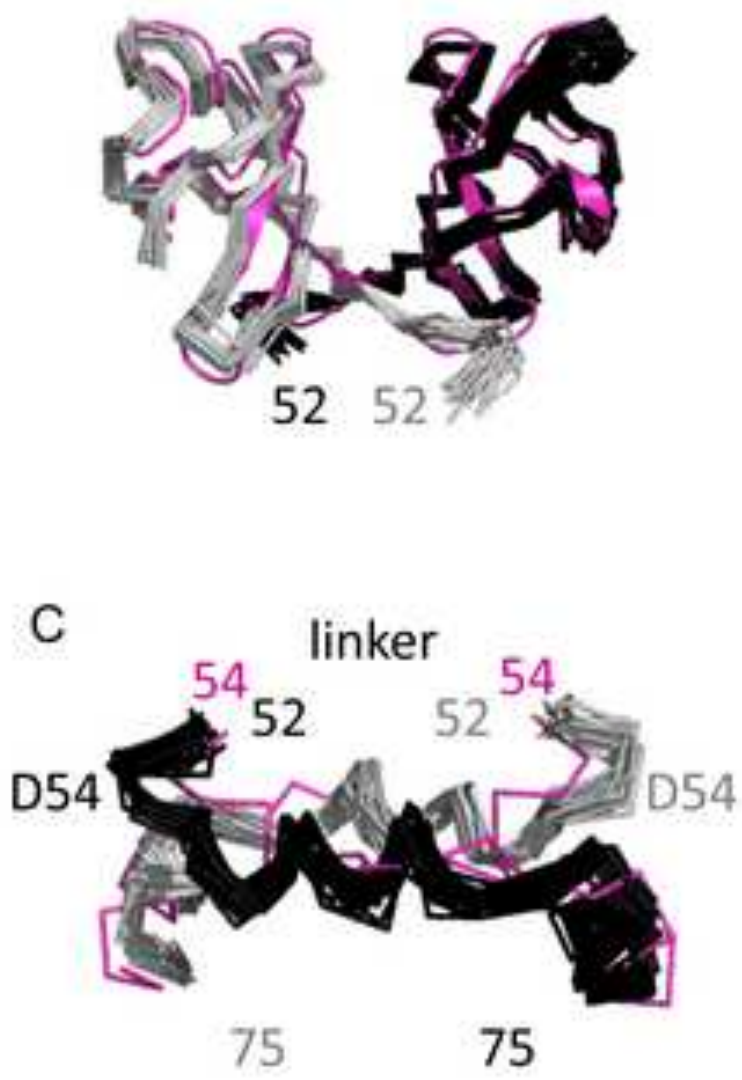

B

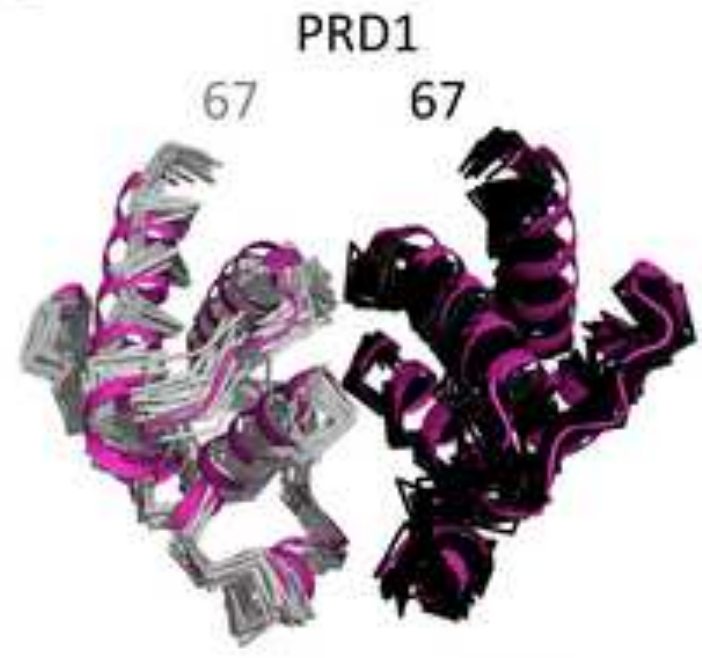

D
167

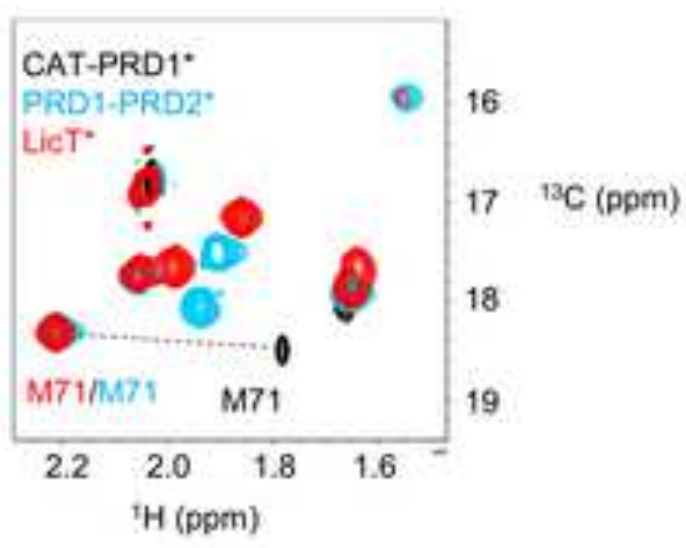

E

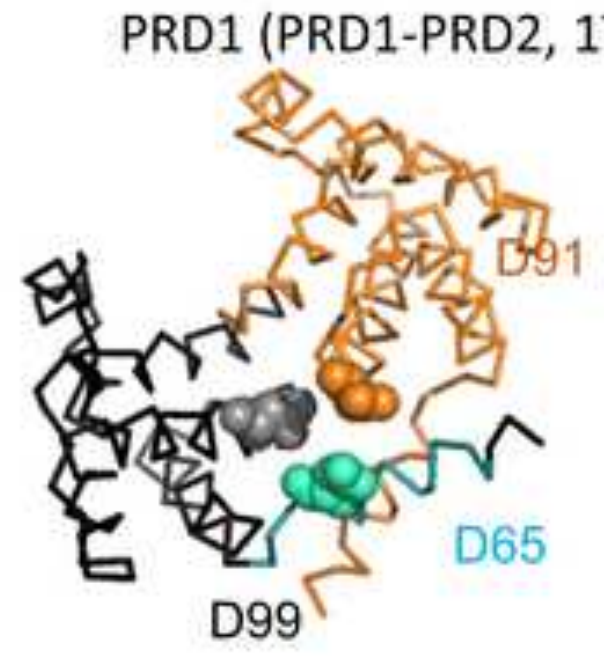

PRD1 (CAT-PRD1*)

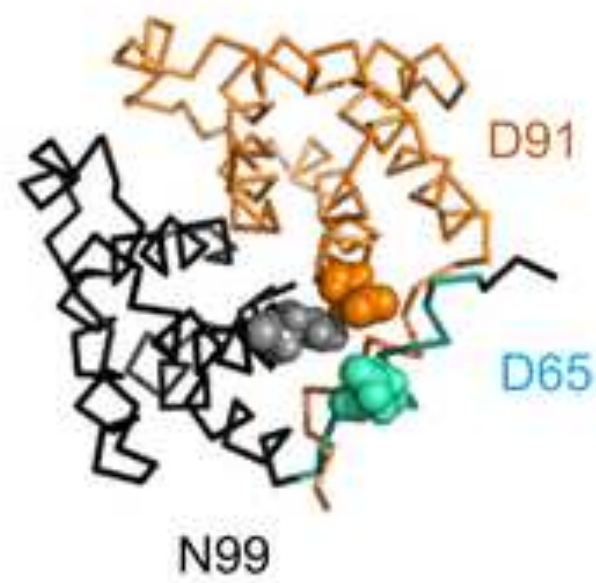

Figure 3, Yang et al. 


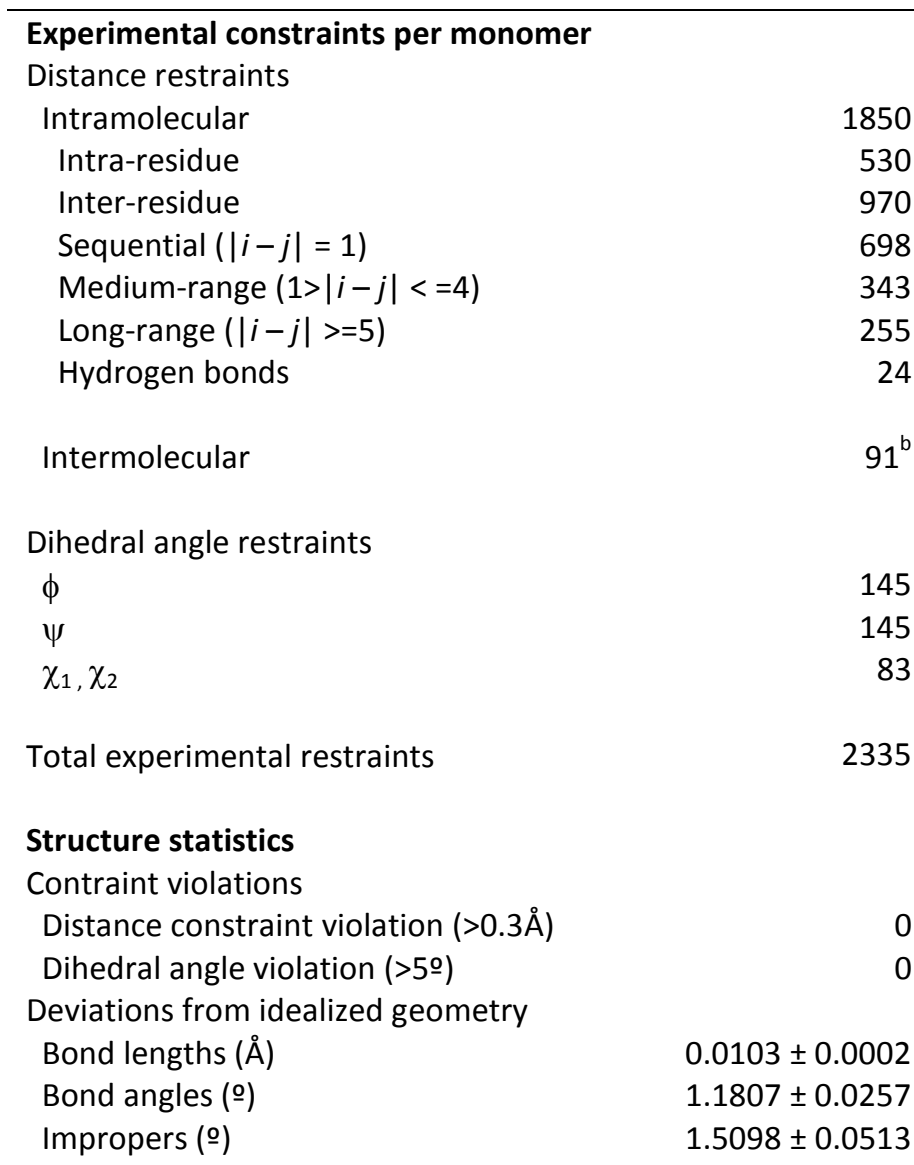

\section{Global quality scores}

Ramachandran statistics ${ }^{\mathrm{a}}(\%)$

Most favoured region 93

Additionally allowed region $\quad 7$

Generously allowed region $\quad 0.1$

Disallowed region 0

Average pairwise r.m.s. deviation** (Å)

A

$1.03 \pm 0.08$

$1.75 \pm 0.11$

$0.58 \pm 0.07$

$1.26 \pm 0.15$

$1.0 \pm 0.09$

$1,61 \pm 0.08$
A+B

Backbone
Heavy
CAT (3-52)
Backbone
Heavy
PRD1 (57-164)
Backbone
Heavy

0

7



Supplementary Material (to Production)
Click here to download Supplementary Material (to Production): Appendix A.docx

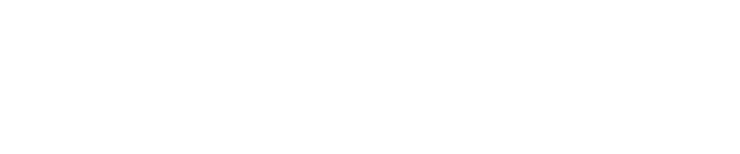

(2) . (1) (1) (1) (1) (1) (1) (1) (1) (1) . . . . . . . . . . 
${ }^{*}$ Conflict of Interest
Click here to downloa

Click here to download Conflict of Interest: declaration-of-competing-interests.docx 